\title{
High accuracy traceable Rutherford backscattering spectrometry of ion implanted samples
}

\author{
J.L. Colaux \& C. Jeynes \\ University of Surrey, Ion Beam Centre, Guildford GU2 7XH, England
}

\begin{abstract}
There are few techniques capable of the non-destructive and model-free measurement at $1 \%$ absolute accuracy of quantity of material in thin films without the use of sample-matched standards. We demonstrate that Rutherford backscattering spectrometry can achieve this robustly, reliably and conveniently. Using $1.5 \mathrm{MeV} \mathrm{He}^{+}$, a $150 \mathrm{keV}$ ion implant into silicon with a nominal fluence of $5 \times 10^{15} \mathrm{As} / \mathrm{cm}^{2}$ has been independently measured repeatedly over a period of 2 years with a mean total combined standard uncertainty of $0.9 \pm 0.3 \%$ relative to an internal standard given by the silicon stopping power (a coverage factor $\mathrm{k}=1$ is used for all uncertainties given). The stopping power factor of this beam in silicon is determined absolutely with a mean total combined standard uncertainty of $0.8 \pm 0.1 \%$, traceable to the $0.6 \%$ uncertainty of the Sb-implanted certified reference material (CRM) from IRMM, Geel. The uncertainty budget highlights the need for the accurate determination of the electronic gain of the detection system and the scattering angle, parameters conventionally regarded as trivial. This level of accuracy is equally applicable to much lower fluences since it is not dominated by any one effect; but it cannot be reached without good control of all of these effects. This analytical method is extensible to non-Rutherford scattering. The stopping power factor of $4.0 \mathrm{MeV}$ lithium in silicon is also determined at $1.0 \%$ absolute accuracy traceable to the Sb-implanted CRM. This work used SRIM2003 stopping powers which are therefore demonstrated correct at $0.8 \%$ for $1.5 \mathrm{MeV} \mathrm{He}$ in $\mathrm{Si}$ and $1 \%$ for $4 \mathrm{MeV} \mathrm{Li}$ in $\mathrm{Si}$.
\end{abstract}

\section{Keywords:}

RBS, EBS, PIXE, Ion beam analysis, Total-IBA, SIMS standards, XRF, Quality Assurance 


\section{Introduction}

The accurate determination of quantity of material is one of the essential motivations of analytical chemistry, and its non-destructive measurement is of great importance in many circumstances, including situations where the sample is intrinsically valuable (e.g. pieces from museum collections ${ }^{1}$ ), or irreplaceable (e.g. forensic samples from crime scenes ${ }^{2}$ ), or where a full characterisation of the sample requires different complementary methods ${ }^{3}$, or where repeated in situ measurements of it are needed ${ }^{4}$. Thin film materials (that is, having surface layers $<\sim 10 \mu \mathrm{m}$ thickness) are ubiquitous in modern technology. For example: semiconductor devices all have thin film active layers, tribological or optical coatings are frequently thin, and interface effects (including corrosion and oxidation) frequently extend beyond the near-surface region.

Some analytical techniques, such as ellipsometry or XRF (X-ray fluorescence), can measure relative thin film thicknesses non-destructively and very precisely, but they depend on assumed models of the sample to obtain absolute values (and when values from XRF are given with high absolute accuracy they also depend on sample-matched standards). Other techniques, such as dynamic SIMS (secondary ion mass spectrometry) can obtain elemental thin film depth profiles with very good depth resolution (destructively, using sputtering), but heavily depend for quantification on sample-matched standards.

Rutherford backscattering spectrometry (RBS) is not deliberately destructive, although the energy deposited by the probing beam usually modifies the sample to at least some extent; we show here that it is capable of an absolute accuracy that is unusually high for thin film characterisation methods, where this accuracy does not depend on any assumptions about the sample, and where no standards related to the sample are required. The method we describe is of broad applicability, valid for a very general class of samples.

RBS has been considered a $1 \%$ technique for the measurement of quantity of material ever since Turkevich made this claim after the Moon landing of Surveyor I in $1966^{5}$. However, the critical demonstration of this claim has proved to be unusually difficult even though the governing equation (Eq.1) is remarkably simple, and in fact has been simplified here without any loss of generality. The signal $A_{C}$ (see Figure 1) is detected from the measurand, $C$, and is given by the product of: the number $Q$ of particles of the probing beam striking the target (containing $C$ ), the number density $f_{C}$ of the measurand present in the target, the probability $\sigma$ of interaction of the beam with the measurand, and the probability of detection $\Omega$ of the reaction product (this is simply the detector solid angle since charged particle detection efficiencies are essentially $100 \%)$ :-

$A_{C}=Q f_{C} \sigma_{C}^{\prime}(E, \theta) \Omega$

$\sigma^{\prime} \equiv \frac{d \sigma}{d \Omega}=\left\{Z_{1} Z_{2} e^{2} \frac{\operatorname{cosec}^{2}(\theta / 2)}{(4 E)}\right\}^{2}$

where $\sigma$ is in this case approximated by the Rutherford differential scattering cross-section ${ }^{6}$ (that is, we assume that point charges $e$ interact in a Coulomb potential) given in Eq.2; this cross-section is a function of the probing beam energy $E$ and the scattering angle $\theta$ as well as 
the atomic numbers $Z_{\mathrm{i}}$ of the beam and target, and is given here for simplicity in the centreof-mass frame of reference.

It is the very simplicity of Eq.1, together with the fact that the interaction cross-section is known analytically, that underlay Turkevich's claim. In principle, the accuracy of this method is important since ion beam analysis of a sample can be effected with multiple detectors of different types, or repeated (since the method is essentially non-destructive) with ion beams of different types or energies. Thus, many different nuclear reaction spectra can be acquired for any particular sample, including RBS, non-Rutherford elastic backscattering (EBS), elastic recoil detection (ERD), or nuclear reaction analysis (NRA) which includes particle-induced $\gamma$-ray emission (PIGE). As well as these data, atomic excitation, that is, particle-induced X-ray emission (PIXE), is always present. All of these together are known as ion beam analysis (IBA) ${ }^{7}$, and the self-consistent treatment of all spectra together is known as "Total-IBA" 8 . Accurate RBS has a wide significance because any Total-IBA analysis inherits the accuracy of the most accurate component of the analysis, and it is quite easy to always include RBS in any Total-IBA measurement. The literature cited earlier ${ }^{1-4}$ all use Total-IBA.

The difficulties of accurately measuring $Q$ and $\Omega$ separately have long been recognised, but are usually sidestepped since they always appear together in the equations. Then any standard sample (for which $f_{C}$ is known) can be used to evaluate the product $Q \Omega$ in Eq.1. This was done by l'Ecuyer et al (1979) ${ }^{9}$ in the classical paper that established the correction to the Rutherford cross-section due to electron screening of the bare nucleus (Coulomb) potential. Their work was nominally at $1.5 \%$ accuracy, but they pointed out that an unaccounted $4 \%$ error remained. The characterisation of a Bi-implanted certified reference material (CRM) was described by Cohen et al ${ }^{10}$ who claimed $1.6 \%$ accuracy (a coverage factor $\mathrm{k}=1$ is used throughout the present paper unless otherwise stated); this was summarised by Davies et al ${ }^{11}$ who underline the problems of inhomogeneity suffered by this "standard", with a fluence range over the whole set of $7 \%$ determined by Wätjen \& Bax ${ }^{12}$ using the classical method of determining thin film thickness by area and weight to provide accurate (certified) standards ${ }^{13}$. In a handbook chapter entitled "Pitfalls", Davies et al ${ }^{14}$ describe this work claiming only $2 \%$. It is worth pointing out that this work was from three groups: Cohen in Paris and Wätjen in Geel used (different) weighed reference standards, whereas the Chalk River group (Davies) made absolute measurements of $Q$ and $\Omega$ independently.

A more accurately certified CRM was established by Ecker et al ${ }^{15}$ who determined the $\mathrm{Sb}$ fluence in Sb-implanted silicon. They found $Q \Omega$ directly from Eq. 1 using a thin evaporated gold film whose thickness was determined traceably by in situ weighing (as for the previous Bi-implanted standards). Their RBS was by the National Metrology Institutes in Berlin and Geel, each having a combined standard uncertainty of $0.9 \%$, but the accurate balance required for traceable quantification is very special equipment (and is now dismantled). The relative uniformity of the implantation was determined separately using very precise XRF. RBS was combined with two other independent techniques, ICP-IDMS (inductively-coupled plasma isotope dilution mass spectrometry) and INAA (instrumental neutron activation analysis), to obtain a combined standard uncertainty for the CRM of $0.6 \%$. 
Jeynes et al ${ }^{16}$ subsequently used the Sb-implanted CRM indirectly to evaluate the uncertainties in an RBS analysis by three labs (Surrey, Budapest \& Lisbon) of an As-implanted sample, demonstrating that, as for the work of the 1980s, each lab could achieve a combined standard uncertainty of about $1 \%$. In the present work we will critically establish the traceability of this method (including a critical and detailed discussion of the uncertainty budget ${ }^{17}$ ), and demonstrate its reliability and generality in practice.

\section{Theory}

\section{Traceability of RBS: Fundamental equations}

The fundamental equations for RBS, together with secondary effects, are discussed at length by Jeynes $e \mathrm{al}^{16}$; for convenience we here briefly review them. Eq.1 implicitly includes an integration of the signal over the thin film thickness: thus, the stopping of the beam in the target is also implicit in this equation since the incident particles lose energy by electronic processes when penetrating the target. This stopping is related to the electron density in the target and is remarkably independent of chemical state: "Bragg's rule" ${ }^{18}$, that the stopping in a compound is simply the linear combination of the stopping in the elements of the compound, is usually a very good approximation. The stopping powers of ion beams in elemental materials are now known fairly well ${ }^{19}$. In this work we do not need measured stopping since we will directly determine the required values with a method traceable to certified reference materials.

However, when depth information about the sample is needed, the stopping power appears at first order. Eq. 3 shows the backscattered particle yield $Y$ (in counts per channel of the analogdigital converter, ADC, used to digitise the energy spectrum seen by the particle detector: see Figure 1) from the surface of a target: the backscattering spectrum will contain partial spectra from each element in the target. At the surface the beam has suffered no energy loss in the target and therefore still has its full energy $E_{0}$.

$Y_{0, C}=Q f_{C} \sigma_{0, C}^{\prime}\left(E_{0}, \theta\right) \Omega \frac{\Delta}{\left[\varepsilon_{0}\right]_{M}^{C}}$

The difference between Eq. 1 and Eq.3 is that the electronic gain $\Delta$ (in keV/channel) is involved in the yield $Y$, where it is not involved in the total number of counts $A$ from a given number of atoms; clearly, the energy loss $\varepsilon$ which governs the shape of the substrate signal (see Figure 1) is also involved. The "stopping cross-section factor" $\left[\varepsilon_{0}\right]_{M}^{C}$, expressed for clarity by Eq. 4 in the "surface energy approximation" ${ }^{20}$, refers to the energy lost by the particle scattered from element $C$ of the matrix $M$.

$$
\begin{aligned}
& {\left[\varepsilon_{0}\right]_{M}^{C}=k_{C} \frac{\varepsilon\left(E_{0}\right)_{M}}{\cos \left(\phi_{1}\right)}+\frac{\varepsilon\left(k_{C} E_{0}\right)_{M}}{\cos \left(\phi_{2}\right)}} \\
& k \equiv \frac{E}{E_{0}}=\left\{\frac{\cos \theta+\left(r^{2}-\sin ^{2} \theta\right)^{1 / 2}}{1+r}\right\}^{2}
\end{aligned}
$$


In Eq.4, $\varepsilon(E)_{M}$ is the energy loss (in $\mathrm{eV} / \mathrm{nm}$ or equivalent units) for a beam of energy $E$ in the matrix $M, \phi_{i}$ are the angles defined by the sample normal and (respectively) the incident or scattered beam directions, and $k_{C}$ is the so-called "kinematical factor" given for each $C$ (in Eq.5) by the conservation of energy and momentum; this expression is valid for any elastic interaction and depends only on the scattering angle and the ratio $r$ of the scattering and scattered nuclear masses (that is, respectively the mass of $\mathrm{C}$ and the mass of the beam particles).

Again, Eq.3 has been simplified without any loss of generality and has been used directly in a hand calculation by Boudreault et al ${ }^{21}$ : an equivalent equation can be given, mutatis mutandis, for the yield $Y_{\mathrm{i}, \mathrm{C}}$ of layer $i$ at any given depth in the material; this is easily calculated by numerical methods, as has been reviewed by Jeynes et al ${ }^{22}$.

\section{Analytical Method}

Eq.1 makes it clear that the quantification of the numbers of counts in a spectrum depends only on the scattering cross-section and the charge-solid-angle product $Q \Omega$. Since the RBS cross-section is derived analytically from the Coulomb potential (Eq.2), the accuracy of the atomic fraction $\left(f_{C}\right)$ determination is given directly by the accuracy of the $Q \Omega$ determination. In fact, the integration implied by Eq.1 does also depend weakly on stopping power, but this is known well enough not to significantly affect the accuracy.

The silicon substrate yield ( $Y$ in Eq.3: see Figure 1) for this beam energy can be used as a well-defined internal standard to determine $Q \Omega$ as proposed by Bianconi et al ${ }^{23}$; this analytical method implies that both the electronic gain $\Delta$ and the stopping cross-section factor $[\varepsilon]$ are accurately known. Jeynes et al ${ }^{16}$ have discussed the accurate measurement of $\Delta$ in detail, but only cite an uncritical determination of $[\varepsilon]^{24}$. We here present a critical determination of $[\varepsilon]$ for $1.5 \mathrm{MeV} \mathrm{He}^{+}$in Si traceable to the Sb-implanted CRM at an accuracy much higher than is available from the SRIM database ${ }^{25}$.

\section{Traceability of RBS: Uncertainty Budget}

Table 1 shows a typical Uncertainty Budget for this analysis which includes a number of secondary effects as well as effects of spectral distortion. Counting statistics $\left(A_{1} \& A_{2}\right)$ should not dominate these types of measurements, and we list ten other effects $\left(A_{3-6} \& B_{1-6}\right)$ most of which are not usually considered when estimating experimental uncertainties in measurements of this type. We discuss these in turn :-

The first thing to consider concerning a signal from a noise-level system is the issue of the signal/noise ratio. The signal background in this case is due to pulse pileup (see Figure 1), which is very roughly linear with counting rate and can never be eliminated. It can be reduced by reducing the count rate, but then the counting time must be increased and questions of cost \& benefit become important. It can be systematically reduced by increasing the time resolution of the pileup rejection circuit, and this would dramatically improve our 
sensitivity. However, in any given experiment the pileup must be modelled, which can be done with various algorithms (see ${ }^{26,27}$ and refs. therein), none of which have any free parameters. That these models are good can be seen from Figure 1. Provided the calculated pileup is well clear of large signals, we estimate that it can be fitted with an accuracy of about $10 \%\left(B_{1}\right.$ in Table 1$)$ : this is because although the model parameters are not strictly free it is not clear precisely what they are, and in practice the modelled pileup is normalised to the signal. Added to this is an extra uncertainty of the model itself $\left(B_{4}\right)$ since the various models give slightly different answers.

The uncertainty in the scattering angle $\left(B_{2}\right)$ affects measured fluence both through the scattering cross-section (Eqs.1\&2) and the exit pathlength in the material (Eqs.3\&4).

The beam energy of electrostatic ion accelerators is usually determined by the energy calibration achieved through standard nuclear techniques such as $(\mathrm{p}, \mathrm{n})$ threshold reactions ${ }^{28}$, resonant $(p, \gamma)$ and $(p, \alpha \gamma)$ nuclear reactions ${ }^{29}$, or direct capture $(p, \gamma)$ reactions ${ }^{30}$. In principle, these high precision techniques lead to a very precise energy calibration (for instance, Demarche et al. claimed a precision of $0.03 \%{ }^{31}$ ). However, to the best of our knowledge, no critical uncertainty budget has yet been assigned to these techniques and their accuracy still needs to be critically addressed in the literature. Moreover, these techniques are quite time consuming due to the low reaction-cross-sections involved ${ }^{30}$, and the necessity of measuring several excitation curves ${ }^{28,29}$. In this work, we took advantage of the ${ }^{16} \mathrm{O}(\alpha, \alpha){ }^{16} \mathrm{O}$ resonance at $3037.9 \pm 2.3 \mathrm{keV}$ to calibrate our accelerator, with the resonance energy determined at $0.08 \%$ from a critical analysis of the literature (see ${ }^{31}$ and refs. therein). The procedure is to measure a $\mathrm{Au} / \mathrm{Ni} / \mathrm{SiO}_{2} / \mathrm{Si}$ calibration sample ${ }^{32}$ at different energies near the resonance: the nominal incident energy is increased from $3020 \mathrm{keV}$ to $3110 \mathrm{keV}$ in $10 \mathrm{keV}$ steps. The actual energy of the incident beam is derived from the peak shape of the ${ }^{16} \mathrm{O}$ signal through the shape of the ${ }^{16} \mathrm{O}(\alpha, \alpha){ }^{16} \mathrm{O}$ cross-section (modelled with appropriate nuclear parameters ${ }^{33}$ ), with a precision of $0.02 \%$ and an accuracy of $0.1 \%$ ( $B_{3}$ in Table 1$)$ dominated by the uncertainty on the energy of the resonance. This same method was used in an approximate way by Andrade et al $^{34}$.

The electronic gain $\Delta$ of the acquisition system as well as the so-called "pulse-height defect" (PHD) of the detector are determined simultaneously from the calibration sample by obtaining the scattered particle energy from the kinematics and using the electronics offset (which is set to zero beforehand) of the acquisition system. It is worth stressing the value of performing the measurements at several incident energies to properly determine the PHD of the detector, which is done here since $3 \mathrm{MeV}$ is used to determine the beam energy. Lennard et al. ${ }^{35}$ also emphasise the distinction between "energy" spectra (for which the PHD is neglected) and pulse-height spectra. Particular attention must be paid to the PHD determination since the electronic gain derived from the data processing is largely related the PHD chosen ${ }^{36}$. The electronics calibration uncertainty $\left(A_{3}\right.$ in Table 1$)$ is evaluated from the standard deviation of the electronic gains $\Delta$ used to properly fit the set of spectra acquired on the calibration sample at various incident energies; in particular, it is the upper limit of the confidence interval (considering $99 \%$ of confidence) given for this standard deviation by the one-tailed probability of the $\chi^{2}$-distribution. 
The simultaneous use of two independent detectors for performing the RBS measurements allows an independent check of the estimates of uncertainty and hence the internal consistency of the calibration parameters (see inset in Figure 1). Moreover, the ratio of the apparent implant fluences measured by each detector should equal unity, with an uncertainty given by:

$U_{\text {Det } A / \text { DetB }}^{2}=\left(U_{1}^{\text {Det A }}\right)^{2}+\left(U_{1}^{\text {Det B }}\right)^{2}$

If this is not the case there must be doubt about the value of the average fluence; the contribution $A_{4}$ reported in the uncertainty budget (Table 1) is an attempt to take account of such a discrepancy:

$A_{4}=\frac{1 / U_{1}^{\text {DetB }}}{1 / U_{1}^{\text {DetA }}+1 / U_{1}^{\text {DetB }}} \times \sqrt{\left(1-\frac{\text { Fluence }_{\text {Det } A}}{\text { Fluence }_{\text {Det } B}}\right)^{2}-\left(U_{\text {DetA } / \text { Det } B}\right)^{2}}$

The first term in Eq.7 is a weighted average of the uncertainties $U_{1}$ : for an unweighted average this term is simply equal to $1 / 2$. The second term of this equation represents the apparent divergence between both detectors taking into account the uncertainty that applies to the ratio Fluence $_{\text {DetA }} /$ Fluence $_{\text {DetB }}$ (Eq.6). We are comparing the difference observed with the most probable expected difference, and estimate the measurement bias simply as a difference of variances. If this difference is negative (giving an imaginary $A_{4}$ ) clearly the difference is less than the expected (most probable) difference - that is, there is no bias. In this case the discrepancy is consistent with what is expected from the statistics. It is worth noting that the apparent disagreement between both detectors $\left(A_{4}\right)$ can easily reach several percent if the electronics are not accurately calibrated.

The uncertainty of the analysis code used (DataFurnace ${ }^{37}$ ) was evaluated as $0.2 \%$ in an IAEA-sponsored intercomparison ${ }^{38}\left(B_{5}\right.$ in Table 1$)$. The uncertainty of the screening correction on the (Rutherford) scattering cross-section (Eq.2) was taken conservatively as the standard deviation of the screening correction factors obtained using the approximations of Molière and Thomas-Fermi ${ }^{9}$ : that is, considering a $1.5 \mathrm{MeV}$ He beam, and using a cover factor $\mathrm{k}=2$ to estimate this important quantity conservatively, we obtain $0.50 \%$ for $\mathrm{Bi}, 0.27 \%$ for $\mathrm{Sb}\left(B_{6}^{\prime}\right.$ in Table 2$)$ and $0.16 \%$ for As $\left(B_{6}\right.$ in Table 1$)$.

The accuracy of the stopping power used to determine the $Q \Omega$ product (through Eq.3) dominates the final uncertainty. Consequently, we have measured the stopping power factor $[\varepsilon]$ for this beam in silicon with the reverse process, that is, using the Sb-CRM to determine the $Q \Omega$ product from Eq.1, and then determining [ $\varepsilon]$ from Eq.3 (see Table 2). The evaluated uncertainty on this determination of $[\varepsilon]$ is $A_{5}$ in Table 1 , which is the uncertainty of the CRM certification $(0.6 \%)$ and $U_{4}^{\prime}$ from Table 2 added in quadrature.

There is also a second-order effect of the stopping power since the Rutherford differential cross-section depends on the energy $E$ of the scattered particles (Eq.2), and therefore the uncertainty on the matrix stopping power will also induce an uncertainty on the probability of a scattering event occurring at a given depth in the material. This extra uncertainty has been evaluated for the particles backscattered by the implanted material at its maximum of distribution $\left(A_{6}\right.$ in Table 1$)$. 


\section{Channelling effects}

We will use the alpha particle backscattering yield from the silicon substrate (see $Y_{0, S i}$ in Figure 1 and discussion of Eq.3) as an internal standard determining $Q \Omega$ in these measurements. But we have to avoid distortion of this yield due to the crystallinity of the silicon: the atoms are very highly correlated to each other and therefore can shadow each other from the highly collimated alpha beam; this is due to the so-called "channelling" effect. Efforts to "randomise" the backscattering yield by choosing pseudo-random directions ${ }^{39}$ or rotating the sample under the beam ${ }^{40}$ are effective only at the $4 \%$ level (see the extended discussion of channelling in Lulli et $a l^{41,42}$ ). For accurate work, amorphisation of crystalline samples is essential to eliminate channelling effects ${ }^{23}$.

\section{Experimental section}

In April 2010, two $100 \mathrm{~mm}$ Si wafers (\#\#20, 21) were amorphised with a (nominally) $3 \times 10^{15} \mathrm{Ar} / \mathrm{cm}^{2}$ implant at $150 \mathrm{keV}$, and subsequently implanted by (nominally) $5 \times 10^{15} \mathrm{As} / \mathrm{cm}^{2}$ at $80 \mathrm{keV}$. The uniformity over the wafers was verified by RBS to be better than $1 \%$, and four-point-probe resistivity measurements on comparable implants (annealed appropriately) show a uniformity of about $0.5 \%$; since this is the precision of the technique, the real uniformity is probably better. The measured Ar fluence was $(3.075 \pm 0.030) \times$ $10^{15} \mathrm{Ar} / \mathrm{cm}^{2}$ where the ratio of Ar content between the two wafers was $1.010 \pm 0.014$. The ratio of As fluences between wafers was determined by RBS as $1.004 \pm 0.003$ : that is, the wafers are indistinguishable. In this work we repeatedly measured samples from wafer \#20 over a period of 2 years.

The Sb-implant CRM is a $15 \mathrm{~mm}$ square piece of the certified reference material IRMM0001/BAML003, provided by the European Institute for Reference Materials and Measurements ${ }^{15}$. This sample was produced by a Sb implantation into a (100) Si wafer, and subsequently amorphised at Surrey to a depth of about $630 \mathrm{~nm}$ with an "Epifab" implant, ${ }^{43}$ that is, a $5 \times 10^{15}{ }^{28} \mathrm{Si} / \mathrm{cm}^{2}$ cold implant at $500 \mathrm{keV}$ on a liquid-nitrogen-cooled stage. Such implants are widely used in defect-engineering applications, and are well known. The implanted fluence of the CRM is $48.1 \times 10^{15} \mathrm{Sb} / \mathrm{cm}^{2}$ certified at an absolute accuracy of $0.6 \%$. The implant was mixed ${ }^{121} \mathrm{Sb}$ and ${ }^{123} \mathrm{Sb}$ such that assuming natural abundance introduces a systematic error of $0.06 \%$ which we neglect.

All RBS measurements were performed on a $2 \mathrm{MV}$ tandem accelerator in a chamber containing a six-axis goniometer ${ }^{44}$ and two "passivated implanted planar silicon" (PIPS) detectors set at $172.8 \pm 0.2^{\circ}$ (DetA) and 148.6 $\pm 0.2^{\circ}$ (DetB) with nominal subtended solid angles of 1.3 and $3.9 \mathrm{msr}$, respectively. The scattering angles were measured directly using a beam-line laser and the six-axis goniometer. The nominal beam size was $2 \mathrm{~mm}$ with a typical beam intensity of 2-25 nA, whatever beam was used $\left(3.0 \mathrm{MeV}{ }^{1} \mathrm{H}^{+}, 1.5 \mathrm{MeV}^{4} \mathrm{He}^{+}, 4.0 \mathrm{MeV}\right.$ ${ }^{7} \mathrm{Li}^{2+}$ or $\left.9.6 \mathrm{MeV}^{12} \mathrm{C}^{4+}\right)$.

For the helium, lithium and carbon RBS measurements, the energy of the incident beam was calculated using an energy calibration factor determined less than three months before 
performing the experiment. For the proton beam measurements, the latest energy calibration factor determined following the ${ }^{16} \mathrm{O}(\alpha, \alpha){ }^{16} \mathrm{O}$ procedure described above was more than 6 months. Consequently, we checked the calibration of our accelerator by using the ${ }^{27} \mathrm{Al}(\mathrm{p}, \gamma){ }^{28} \mathrm{Si},{ }^{13} \mathrm{C}(\mathrm{p}, \gamma){ }^{14} \mathrm{~N}$ and ${ }^{32} \mathrm{~S}(\mathrm{p}, \gamma){ }^{33} \mathrm{Cl}$ nuclear resonances occurring respectively at $(991.9 \pm 0.1),(1747.6 \pm 0.9)$ and $(3379 \pm 1) \mathrm{keV}^{45}$. The stability of the energy calibration factor, over this period of six months, was found to be better than $0.04 \pm 0.12 \%$.

The experimental conditions used for the analysis of the Sb-implant CRM and the As-implant samples are reported in Table 3. All spectra analysed in Surrey used the DataFurnace software (NDF version 9.4h ${ }^{46}$ ). The electronic gain and PHD of both detectors were fitted from the calibration sample $\left(\mathrm{Au} / \mathrm{Ni} / \mathrm{SiO}_{2} / \mathrm{Si}\right)$ spectra collected at various incident energies ${ }^{32}$, ${ }^{47}$ by assuming a linear calibration with a null offset, which was initially verified using a high precision electronic pulser. The Molodtsov \& Gurbich pileup correction ${ }^{26}$ was used together with the pileup rejection capability of the shaping amplifiers. The electron screening factors given by Andersen et $^{4}{ }^{48}$ were used.

\section{Results}

The As-implant sample has been independently measured repeatedly over a period of 2 years using a ${ }^{4} \mathrm{He}^{+}$beam at $1.5 \mathrm{MeV}$ and the $\mathrm{Si}$ substrate yield as an internal standard. A typical uncertainty budget obtained for an As fluence determination using this ion beam is reported in Table 1, and the measured fluences with their uncertainties are tabulated in Table 4 and shown as a function of time in Figure 2. The error bars given in this figure are the "reduced uncertainty": this is, for each measurement, the total combined standard uncertainty (penultimate column of Table 4) less the contribution of all systematic errors (i.e. $B_{3-6} \& A_{5-6}$ ) which are identical for each dataset measured with the $1.5 \mathrm{MeV} \mathrm{He}^{+}$beam. The reduced uncertainties were used to calculate the weighted average, $(4.647 \pm 0.014) \times 10^{15} \mathrm{As} / \mathrm{cm}^{2}$, also shown in Figure 2.

The Sb-implanted CRM was measured similarly, to derive the uncertainty of the stopping power factor for $\mathrm{Si}$ " $[\varepsilon]_{S i}$ " from the certified $\mathrm{Sb}$ fluence. The results are shown in Table 4. The uncertainty budget is constructed similarly to Table 1 and is shown in Table 2; the total combined uncertainty $U_{4}^{\prime}$ from Table 2 is therefore combined with the uncertainty of the CRM certification $(0.6 \%)$ to evaluate the uncertainty on $[\varepsilon]_{S i}$ used in Table $1\left(A_{5}\right)$.

Table 4 also tabulates the measured fluences with their uncertainties for $\mathrm{H}, \mathrm{Li}$ and $\mathrm{C}$ beams, which are shown in Figure 3. In this case, the error bars represent the total combined standard uncertainty $\left(\mathrm{U}_{4}\right.$ in Table 4$)$ since we are comparing the results obtained with different ion beams. The combined uncertainty of the weighted average obtained for the $1.5 \mathrm{MeV}$ alpha beam measurements is $0.9 \%$. 


\section{Discussion}

\section{Stopping power factor}

The uncertainty of the silicon stopping power factor for the $1.5 \mathrm{MeV}^{4} \mathrm{He}^{+}$beam $\left(A_{5}\right.$ and see Table 2) was critically estimated through the Sb-implant CRM analysis. The measured $\mathrm{Sb}$ fluence was found to be $48.0 \times 10^{15} \mathrm{Sb} / \mathrm{cm}^{2}$ with a standard uncertainty of $0.52 \%\left(U_{4}^{\prime}\right.$ in Table 2) derived from our uncertainty budget disregarding the silicon stopping power uncertainties $\left(A_{5}^{\prime} \& A_{6}^{\prime}\right)$. The ratio between the certified and measured Sb fluences is therefore equal to 1.002 with an uncertainty of $0.8 \%$ derived from the combination of the uncertainties that apply to the measured $(0.52 \%)$ and certified $(0.6 \%)$ values. Since this ratio is closer to unity $(0.2 \%)$ than its combined uncertainty $(0.8 \%)$, we can conclude that the error on $[\varepsilon]$ (obtained from the SRIM2003 database) is lower than $0.8 \%$ ( $A_{5}$ in Table 1), which is about 3 times lower than the uncertainty estimated from the experimental measurements of $[\varepsilon]$ for this target and beam (see the SRIM and other databases ${ }^{25,49}$ ).

This method measures the stopping power factor for a material directly for a particular beam and can be used for any beam and any material. Similar measurements were made for the $4 \mathrm{MeV} \mathrm{Li} \mathrm{beam,} \mathrm{again} \mathrm{finding} \mathrm{that} \mathrm{the} \mathrm{SRIM2003} \mathrm{database} \mathrm{was} \mathrm{accurate} \mathrm{(this} \mathrm{time} \mathrm{at} \mathrm{1 \% ).}$ If measurements are made at a series of beam energies, then the stopping power itself can be extracted (see Barradas et al ${ }^{50}$ for example). This has not yet been done, but is clearly feasible at accuracies much higher than are possible with direct methods.

\section{Robustness of the analytical method}

The relative uncertainty of the average of 2 detectors $\left(U_{2}\right.$ in Table 1$)$ is dominated by the counting statistics in the case shown, but it is easy to count longer so that no one effect dominates the uncertainty. We deliberately have detectors with significantly different solid angles so that with greatly different counting rates (and consequently with different counting statistics and pileup background) the effect that dominates the combined uncertainty differs between detectors. For much smaller fluences (the Surrey implantation QA protocol uses $1 \times 10^{15} \mathrm{As} / \mathrm{cm}^{2}$ ) the dominating effects are usually pileup for the large detector and counting statistics for the small one. Note that Table 2 shows that for some cases the uncertainty from the scattering angle dominates: we have reached an accuracy for which there are many similarly sized contributions to the uncertainty. It will not be easy to improve significantly on this.

Therefore, using two detectors gives robustness to the method since, apart from having two independent data channels which ought to agree, we also have data channels which should be subject to different types of uncertainty. The method consequently has in-built selfconsistency checks which should be able to signal the presence of extra error.

Figure 2 shows that each measurement agrees with the weighted mean within the error bars given by the reduced uncertainty $\left(U_{5}\right.$, see Table $\left.1 ; \mathrm{k}=1\right)$ except the second point of measurement performed in June 13 that requires one to consider a coverage factor $k=2$. This 
is nevertheless expected since the reduced uncertainty is largely dominated by the counting statistics on the implant signal. The weighted mean presented in this work $(4.647 \pm 0.014$ $10^{15} \mathrm{at} / \mathrm{cm}^{2}$ ) is about $1.2 \%$ higher than the average reported by Jeynes et al. ${ }^{16}$. We believe that this difference, if indeed a difference equivalent to $\mathrm{k}=1.2$ is significant, is mainly related to the electronic gain determination which directly changes the As-fluence measured through the a-Si yield (see Eq.3). We have reprocessed the Surrey data used previously ${ }^{16}$ by paying extra attention to the PHD and electronics gain determination, finding a value of $(4.632 \pm 0.029) 10^{15} \mathrm{As} / \mathrm{cm}^{2}$ which is in much better agreement with the weighted means presented both in this and the previous work. This shows how difficult it is to determine the electronics gain with accuracy better than $0.5 \%$ : in particular, the determination of the PHD and the electronics offset is crucial for that level of precision. A careful processing of the spectra acquired on the $\mathrm{Au} / \mathrm{Ni} / \mathrm{SiO}_{2} / \mathrm{Si}$ sample at various incident energies (see calibration method described above) nevertheless allows one to determine these parameters with that level of accuracy. The details of the procedure required will be published elsewhere.

\section{Generalisation to other ion beams}

The uncertainty budget reported in Table 1 for the $1.5 \mathrm{MeV}{ }^{4} \mathrm{He}^{+}$beam can be easily extended to any other ion beam. Indeed, only the contributions $A_{5}$ and $A_{6}$ are directly related to the ion beam properties (i.e. energy and ion type) through the uncertainty in the silicon stopping power factor. This stopping factor can always be estimated from the semi-empirical data compilations ${ }^{25,49}$, but where it can be determined directly from the CRM the uncertainty can be dramatically reduced. For heavier beams the screening correction on the Rutherford scattering cross-section is larger but still known to a good approximation, with an uncertainty $\left(B_{6}\right)$ directly deduced from the comparison between the screening factors obtained in the Molière and Thomas-Fermi approximations ${ }^{9}$. The very good agreement between the measurements performed with $1.5 \mathrm{MeV}^{4} \mathrm{He}^{+}, 4.0 \mathrm{MeV}^{7} \mathrm{Li}^{2+}$ and $9.6 \mathrm{MeV}{ }^{12} \mathrm{C}^{4+}$ is shown in Figure 3. For the lithium beam the stopping power factor is evaluated directly from the CRM with an uncertainty of $1 \%$ and an Uncertainty Budget directly comparable to Table 2; but for the carbon beam the uncertainty in the stopping power factor is evaluated directly from Helmut Paul's compilation ${ }^{49}$.

For higher energy beams the Rutherford approximation breaks down. Non-Rutherford scattering needs a full quantum mechanical treatment to harmonise datasets of measured cross-sections (which are often strong functions of scattering angle), and determining the uncertainty (which may be large) of such "evaluated" cross-section datasets is difficult ${ }^{51}$ and has not been done for the evaluated scattering cross-sections for $\mathrm{H}$ on silicon ${ }^{52}$. We expect that this uncertainty accounts for the very large observed discrepancy $(17.3 \pm 1.6 \%)$ between the certified and the measured $\mathrm{Sb}$ fluence of the Sb-CRM when measured with $3.0 \mathrm{MeV}{ }^{1} \mathrm{H}^{+}$ even though the measurement is precise (with a small uncertainty of $1.3 \%$ ) with the expected error in the silicon stopping power evaluated by SRIM03 is expected to be quite small. For this non-Rutherford case the uncertainty assigned to the cross-section $\left(B_{6}\right)$ is entirely different from the Rutherford case: but the other uncertainties are also evaluated quite differently. Since the kinematics are different (see Eq.5) the elemental edges are much closer together in 
energy, affecting both the pileup background $\left(B_{1}\right)$ and the pileup model $\left(B_{4}\right)$. The variation of cross-section with angle $\left(B_{2}\right)$ is a different function, and the code uncertainty is also larger $\left(B_{5}\right)$. All of these effects are outside the scope of the present work. Here we restrict ourselves to pointing out that the method remains valid, but the construction of the Uncertainty Budget is very considerably harder.

\section{Future developments}

The present method depends for its traceable accuracy on an internal standard from which the $Q \Omega$ product can be obtained, where this internal standard is independently determined absolutely. Here we have relied on the silicon stopping which has been measured traceable to the Sb-implanted CRM.

However, in principle, measured charge $(Q)$ could be used directly as the reference quantity if it could be determined sufficiently accurately, a problem on which there has been relatively little critical published work so far. It is not easy to measure charged particle beam fluences at sub-1\% absolute accuracy because the electronic environment of the scattering chamber is very complex. This is very widely acknowledged in the ion implantation community: even research implanters with relatively low beam currents, simple beam lines and comprehensive instrumentation cannot currently be specified at better than $2 \%$ (and that is challenging!), and Gries' suggestion of "Quantitative Ion-Implantation" ${ }^{53}$ has not been taken up in practice. Nevertheless, it is possible to determine $Q$ at quite high accuracy by a variety of methods, and it may even be practical to directly determine the ion beam current with intrinsically traceable methods based on SQUIDs ${ }^{54}$. 


\section{Conclusion}

In this work, we have demonstrated, by performing multiple independent measurements of an implanted sample over a period of 2 years, that the implant fluence can be reliably determined at a traceable accuracy of about $1 \%$ for reasonable acquisition times $(\mathrm{Q} \cong 30 \mu \mathrm{C})$, and that the measured fluences have the agreement expected from the estimated global uncertainty. Moreover, measurement precision (for extended measurement times) has been demonstrated at $0.3 \%$, showing that the accuracy is currently limited by the uncertainty in stopping power used (or, equivalently, by the uncertainty in the measurement of the chargesolid-angle product).

This method has a general applicability: the same measurement protocol was successfully applied to various ion beams, and comparable methods can be constructed for almost any type of sample, although $1 \%$ accuracy is not always available.

Whatever the incident beam, it is clear that the uncertainty of the material stopping power usually dominates the best accuracy of this analytical method. In the present work, we have critically determined this uncertainty for a helium beam and silicon-based samples essentially by directly measuring the stopping power factor using an Sb-implanted silicon sample certified at $0.6 \%$, with a consequent uncertainty on the measured fluence in this case of $0.8 \%$. This direct stopping power measurement method can be extended to any ion-matter couple, provided that appropriate certified reference materials are available.

It should be emphasised that we have here substituted traceability based on the stopping power factor (a physical constant) for traceability based on an artefact (the Sb-implanted CRM). By determining the stopping power factor (through the CRM) we have dramatically generalised the availability and usability of this particular measurement protocol, since although creating and certifying a CRM is very difficult, anyone with an ion implanter can make an amorphised silicon wafer for themselves.

In general, of the non-destructive thin film characterisation techniques available, ion beam analysis (including RBS) is the most quantitative (that is, traceably accurate) while not requiring either sample-matched standards or further information about the sample. All of the many and very powerful methods available today have their own strengths, and modern materials analysis should make full use of the toolbox of complementary techniques; Total-IBA has a significant place in this toolbox. 
1. L. de Viguerie, L. Beck, J. Salomon, L. Pichon and P. Walter, "Composition of Renaissance Paint Layers: Simultaneous Particle Induced X-ray Emission and Backscattering Spectrometry", Anal Chem, 2009, 81, 7960-7966

2. M. E. Christopher, J.-W. Warmenhoeven, F. S. Romolo, M. Donghi, R. P. Webb, C. Jeynes, N. I. Ward, K. J. Kirkby and M. J. Bailey, "A New Quantitative Method for Gunshot Residue Analysis by Ion Beam Analysis", Analyst, in press, DOI: 10.1039/C1033AN00597F

3. D. Krecar, M. Rosner, M. Draxler, P. Bauer and H. Hutter, "Quantitative analysis of the Ge concentration in a SiGe quantum well: comparison of low-energy RBS and SIMS measurements", Anal Bioanal Chem, 2006, 384, 525-530

4. J. Demeulemeester, D. Smeets, C. M. Comrie, C. Van Bockstael, W. Knaepen, C. Detavernier, K. Temst and A. Vantomme, "The influence of Pt redistribution on Ni(1-x)Pt(x)Si growth properties", J Appl Phys, 2010, 108, 043505.

5. A. L. Turkevich, E. J. Franzgrote and J. H. Patterson, "Chemical Analysis of Moon at Surveyor V Landing Site", Science, 1967, 158, 635-637

6. E. Rutherford, "The Scattering of alpha and beta Particles by Matter and the Structure of the Atom", Philos Mag, 1911, 21, 669-688

7. C. Jeynes, R. P. Webb and A. Lohstroh, "Ion Beam Analysis: A Century of Exploiting the Electronic and Nuclear Structure of the Atom for Materials Characterisation", Reviews of Accelerator Science and Technology, 2011, 04, 41-82

8. C. Jeynes, M. J. Bailey, N. J. Bright, M. E. Christopher, G. W. Grime, B. N. Jones, V. V. Palitsin and R. P. Webb, "'Total IBA" - Where are we?", Nucl Instrum Meth B, 2012, 271, 107-118

9. J. L'Ecuyer, J. A. Davies and N. Matsunami, "How Accurate Are Absolute Rutherford Backscattering Yields", Nucl Instrum Methods, 1979, 160, 337-346

10. C. Cohen, J. A. Davies, A. V. Drigo and T. E. Jackman, "Intercomparison of Absolute Standards for RBS Studies", Nuclear Instruments \& Methods in Physics Research, 1983, 218, 147-148

11. J. A. Davies, T. E. Jackman, H. L. Eschbach, W. Dobma, U. Wätjen and D. Chivers, "Calibration of the Harwell Series-li Bi-Implanted Rbs Standards", Nucl Instrum Meth B, 1986, 15, 238-240

12. U. Wätjen and H. Bax, "Bi-Implanted Silicon Reference Material Revisited - Uniformity of the Remaining Batch", Nucl Instrum Meth B, 1994, 85, 627-632

13. U. Wätjen, H. Bax and P. Rietveld, "Evaporated and Implanted Reference Layers for Calibration in Surface-Analysis", Surf Interface Anal, 1992, 19, 253-258

14. J. A. Davies, W. N. Lennard and I. V. Mitchell, in Handbook of Ion Beam Materials Analysis, eds. J. R. Tesmer and M. Nastasi, Materials Research Society, Pittsburgh, Editon edn., 1992.

15. K. H. Ecker, U. Wätjen, A. Berger, L. Persson, W. Pritzkow, M. Radtke and H. Riesemeier, "RBS, SY-XRF, INAA and ICP-IDMS of antimony implanted in silicon - A multi-method approach to characterize and certify a reference material", Nucl Instrum Meth B, 2002, 188, 120-125

16. C. Jeynes, N. P. Barradas and E. Szilágyi, "Accurate Determination of Quantity of Material in Thin Films by Rutherford Backscattering Spectrometry", Anal Chem, 2012, 84, 6061-6069

17. K. A. Sjöland, F. Munnik and U. Wätjen, "Uncertainty budget for ion beam analysis", Nucl Instrum Meth B, 2000, 161, 275-280

18. W. H. Bragg and M. A. Elder, "On the alpha particles of radium, and their loss of ranae in passing through various atoms and molecules.", Philos Mag, 1905, 10, 318-340 
19. J. F. Ziegler, M. D. Ziegler and J. P. Biersack, "SRIM - The stopping and range of ions in matter (2010)", Nucl Instrum Meth B, 2010, 268, 1818-1823

20. W. K. Chu, J. W. Mayer and M. A. Nicolet, "Backscattering Spectrometry", Academic Press: New York, 1978

21. G. Boudreault, C. Jeynes, E. Wendler, A. Nejim, R. P. Webb and U. Wätjen, "Accurate RBS measurement of ion implant doses in a silicon", Surf Interface Anal, 2002, 33, 478-486

22. C. Jeynes, N. P. Barradas, P. K. Marriott, G. Boudreault, M. Jenkin, E. Wendler and R. P. Webb, "Elemental thin film depth profiles by ion beam analysis using simulated annealing - a new tool", J Phys D Appl Phys, 2003, 36, R97-R126

23. M. Bianconi, F. Abel, J. C. Banks, A. C. Font, C. Cohen, B. L. Doyle, R. Lotti, G. Lulli, R. Nipoti, I. Vickridge, D. Walsh and E. Wendler, "The Si surface yield as a calibration standard for RBS", Nucl Instrum Meth B, 2000, 161, 293-296

24. N. P. Barradas, K. Arstila, G. Battistig, M. Bianconi, N. Dytlewski, C. Jeynes, E. Kotai, G. Lulli, M. Mayer, E. Rauhala, E. Szilágyi and M. Thompson, "Summary of "IAEA intercomparison of IBA software"', Nucl Instrum Meth B, 2008, 266, 1338-1342

25. J. F. Ziegler, "SRIM website", http://www.srim.org/

26. S. L. Molodtsov and A. F. Gurbich, "Simulation of the pulse pile-up effect on the pulse-height spectrum", Nucl Instrum Meth B, 2009, 267, 3484-3487

27. N. P. Barradas and M. A. Reis, "Accurate calculation of pileup effects in PIXE spectra from first principles", X-Ray Spectrom, 2006, 35, 232-237

28. R. O. Bondelid and E. E. D. Whiting, "(p,n) Threshold-Curve Shapes and Measurements of Threshold Energy with ${ }^{1} H^{+}$Beams", Phys Rev B, 1964, 134, B591-B594

29. C. Jeynes and N. P. Barradas, in Handbook of Modern Ion Beam Materials Analysis, ed. Y. W. a. M. Nastasi, Materials Research Society, Warrendale, Pennsylvania, Editon edn., 2009, pp. 347-383.

30. C. Rolfs, W. S. Rodney, S. Durrance and H. Winkler, "New Method for Measurement of Proton-Beam Energies", Nucl Phys A, 1975, A240, 221-234

31. J. Demarche and G. Terwagne, "Precise measurement of the differential cross section from the ${ }^{16} \mathrm{O}(\alpha, \alpha)^{16} \mathrm{O}$ elastic reaction at 165 degrees and 170 degrees between 2.4 and $6.0 \mathrm{MeV}$ ", J Appl Phys, 2006, 100, 124909.

32. C. Jeynes, N. P. Barradas, M. J. Blewett and R. P. Webb, "Improved ion beam analysis facilities at the University of Surrey", Nucl Instrum Meth B, 1998, 136, 1229-1234

33. A. F. Gurbich, "Evaluation of non-Rutherford proton elastic scattering cross section for oxygen", Nucl Instrum Meth B, 1997, 129, 311-316

34. E. Andrade, C. Solis and M. F. Rocha, "Application of the SIMNRA code to calibrate the analyzing magnet associated with a Van de Graaff accelerator", Nucl Instrum Meth B, 2007, 261, 553-556

35. W. N. Lennard, H. Xia and J. K. Kim, "Revisiting the stopping powers of Si and SiO2 for He-4 ions: 0.5-2.0 MeV", Nucl Instrum Meth B, 2004, 215, 297-307

36. A. F. Gurbich and C. Jeynes, "Evaluation of non-Rutherford proton elastic scattering crosssection for magnesium", Nucl Instrum Meth B, 2007, 265, 447-452

37. N. P. Barradas, C. Jeynes and R. P. Webb, "Simulated annealing analysis of Rutherford backscattering data", Appl Phys Lett, 1997, 71, 291-293 
38. N. P. Barradas, K. Arstila, G. Battistig, M. Bianconi, N. Dytlewski, C. Jeynes, E. Kotai, G. Lulli, M. Mayer, E. Rauhala, E. Szilágyi and M. Thompson, "International Atomic Energy Agency intercomparison of ion beam analysis software", Nucl Instrum Meth B, 2007, 262, 281-303

39. A. L'Hoir, D. Schmaus, J. Cawley and O. Jaoul, "Depth Profiling Light-Nuclei in Single-Crystals a Combined Nuclear-Reaction and Rbs Technique to Minimize Unwanted Channeling Effects", Nuclear Instruments \& Methods in Physics Research, 1981, 191, 357-366

40. M. Kokkoris, A. Spyrou, G. Perdikakis, R. Vlastou, C. T. Papadopoulos, A. Lagoyannis, E. Simoen and S. Kossionides, "On the radiation damage effects in semiconductors beyond the end of range of implanted protons at high energies and fluences", Nucl Instrum Meth B, 2005, 240, 168-173

41. G. Lulli, E. Albertazzi, M. Bianconi, R. Nipoti, M. Cervera, A. Camera and C. Cellini, "Stopping and damage parameters for Monte Carlo simulation of MeV implants in crystalline Si", J Appl Phys, 1997, 82, 5958-5964

42. G. Lulli, E. Albertazzi, M. Bianconi, G. G. Bentini, R. Nipoti and R. Lotti, "Determination of He electronic energy loss in crystalline Si by Monte-Carlo simulation of Rutherford backscattering-channeling spectra", Nucl Instrum Meth B, 2000, 170, 1-9

43. J. P. Zhang, R. J. Wilson, P. L. F. Hemment, A. Claverie, F. Cristiano, P. Salles, J. Q. Wen, J. H. Evans, A. R. Peaker and G. J. Parker, "Regrowth Behavior of $\mathrm{Si}_{1-\mathrm{X}} \mathrm{G} e_{\chi} / \mathrm{Si}$ Structures Formed by Ge+ Ion-Implantation and Post Amorphization", Nucl Instrum Meth B, 1994, 84, 222-228

44. A. Simon, C. Jeynes, R. P. Webb, R. Finnis, Z. Tabatabian, P. J. Sellin, M. B. H. Breese, D. F. Fellows, R. van den Broek and R. M. Gwilliam, "The new Surrey ion beam analysis facility", Nucl Instrum Meth B, 2004, 219, 405-409

45. J. B. Marion, "Accelerator Calibration Energies", Rev Mod Phys, 1966, 38, 660-668

46. N. P. Barradas and C. Jeynes, "Advanced physics and algorithms in the IBA DataFurnace", Nucl Instrum Meth B, 2008, 266, 1875-1879

47. C. Pascual-Izarra and N. P. Barradas, "Introducing routine pulse height defect corrections in IBA", Nucl Instrum Meth B, 2008, 266, 1866-1870

48. H. H. Andersen, F. Besenbacher, P. Loftager and W. Moller, "Large-Angle Scattering of Lightlons in the Weakly Screened Rutherford Region", Phys Rev A, 1980, 21, 1891-1901

49. P. Helmut, "Stopping Power for Light lons", http://www.exphys.unilinz.ac.at/stopping/stopp bot.htm

50. N. P. Barradas, E. Alves, Z. Siketić and I. Bogdanović Radović, "Stopping power of He, C and O in TiO2", Nucl Instrum Meth B, 2012, 273, 22-25

51. E. V. Gai and A. F. Gurbich, "Evaluated C-12(He-4,He-4)C-12 cross-section and its uncertainty", Nucl Instrum Meth B, 2013, 296, 87-91

52. A. F. Gurbich, "Evaluated differential cross-sections for IBA", Nucl Instrum Meth B, 2010, 268, $1703-1710$

53. W. H. Gries, "Quantitative Ion-Implantation - Theoretical Aspects", Int J Mass Spectrom, $1979,30,97-112$

54. L. Hao, J. C. Macfarlane, C. Carr and J. C. Gallop, "HTS current concentrator for remote sensing of charged particle beams", leee T App/ Supercon, 2003, 13, 739-742

55. Guide to the Expression of Uncertainty in Measurement, International Organization for Standardization, Geneva, Switzerland, 1995. 


\section{Tables}

Table 1: Uncertainty budget obtained for the fluence determination of the As-implant sample using a ${ }^{4} \mathrm{He}^{+} 1.5 \mathrm{MeV}$ ion beam $(Q=40 \mu \mathrm{C})$.

\begin{tabular}{|c|c|c|c|c|}
\hline & Type & Det A & & Det B \\
\hline Pileup correction (As signal), $\%$ & & 0.3 & & 0.8 \\
\hline Pileup uncertainty ( $10 \%$ of total correction), $\%$ & $\mathrm{~B}_{1}$ & 0.03 & & 0.08 \\
\hline Counting statistics, implant signal, $\%$ & $\mathrm{~A}_{1}$ & 1.60 & & 0.85 \\
\hline Counting statistics, a-Si signal, $\%$ & $\mathrm{~A}_{2}$ & 0.25 & & 0.13 \\
\hline Scattering angle $\sim 1 / \sin ^{4}(\theta / 2)$ and $1 / \cos (\theta), \%$ & $\mathrm{~B}_{2}$ & 0.08 & & 0.41 \\
\hline Electronics calibration uncertainty, $\%$ & $\mathrm{~A}_{3}$ & 0.26 & & 0.14 \\
\hline Relative uncertainty (dataset), $\%$ & $\mathrm{U}_{1}$ & 1.64 & & 0.97 \\
\hline Relative uncertainty of average of 2 detectors (dataset), $\%$ & $\mathrm{U}_{2}$ & & 0.83 & \\
\hline Beam energy, $\%$ & $\mathrm{~B}_{3}$ & & 0.20 & \\
\hline Disagreement between both detectors, $\%$ & $\mathrm{~A}_{4}$ & & 0.00 & \\
\hline Pileup uncertainty (from model), $\%$ & $\mathrm{~B}_{4}$ & & 0.20 & \\
\hline Code uncertainty, $\%$ & $\mathrm{~B}_{5}$ & & 0.20 & \\
\hline Rutherford cross-section (screening uncertainty), $\%$ & $\mathrm{~B}_{6}$ & & 0.16 & \\
\hline Si stopping power (uncertainty on the a-Si yield), $\%$ & $\mathrm{~A}_{5}$ & & 0.80 & \\
\hline Si stopping power (uncertainty on the implant counts), $\%$ & $\mathrm{~A}_{6}$ & & 0.03 & \\
\hline Combined extra systematic uncertainty, $\%$ & $\mathrm{U}_{3}$ & & 0.89 & \\
\hline Total combined standard uncertainty (accuracy), $\%$ & $\mathrm{U}_{4}$ & & 1.22 & \\
\hline Reduced uncertainty & $\mathrm{U}_{5}$ & & 0.83 & \\
\hline
\end{tabular}

$U_{1}^{2}=\sum_{i=1}^{3} A_{i}^{2}+\sum_{i=1}^{2} B_{i}^{2} ; U_{2}^{-2}=U_{1 A}^{-2}+U_{1 B}^{-2} ; U_{3}^{2}=\sum_{i=4}^{6} A_{i}^{2}+\sum_{i=3}^{6} B_{i}^{2} ; U_{4}^{2}=U_{2}^{2}+U_{3}^{2}$

Measurement uncertainties of Type A (statistical estimate available) and Type B (estimate made by other methods) are defined in GUM ${ }^{55}$, and see ${ }^{17}$. See text for discussion of $A_{i}$ and $\mathrm{B}_{\mathrm{i}}$. The reduced uncertainty ( $U_{5}$; this is the measurement precision shown in Figure 2 ) for a given beam is given by $U_{4}$ less the systematic contributions $\left(B_{3-6} \& A_{5-6}\right)$ in quadrature. 
Table 2: Uncertainty budget obtained for the fluence determination of the Sb-implanted CRM using a ${ }^{4} \mathrm{He}^{+} 1.5 \mathrm{MeV}$ ion beam $(\mathrm{Q}=300 \mu \mathrm{C})$.

\begin{tabular}{|c|c|c|c|c|}
\hline & Type & Det A & & Det B \\
\hline Pileup correction (Sb signal), \% & & 0.16 & & 0.36 \\
\hline Pileup uncertainty (10\% of total correction), $\%$ & $\mathrm{~B}_{1}^{\prime}$ & 0.02 & & 0.04 \\
\hline Counting statistics, implant signal, $\%$ & $\mathrm{~A}_{1}^{\prime}$ & 0.12 & & 0.06 \\
\hline Counting statistics, a-Si signal, $\%$ & $\mathrm{~A}_{2}^{\prime}$ & 0.08 & & 0.05 \\
\hline Scattering angle $\sim 1 / \sin ^{4}(\theta / 2)$ and $1 / \cos (\theta), \%$ & $\mathrm{~B}_{2}^{\prime}$ & 0.07 & & 0.40 \\
\hline Electronics calibration uncertainty, $\%$ & $\mathrm{~A}_{3}^{\prime}$ & 0.29 & & 0.17 \\
\hline Relative uncertainty (dataset), \% & $\mathrm{U}_{1}^{\prime}$ & 0.33 & & 0.44 \\
\hline Relative uncertainty of average of 2 detectors (dataset), $\%$ & $\mathrm{U}_{2}^{\prime}$ & & 0.27 & \\
\hline Beam energy, $\%$ & $\mathrm{~B}_{3}^{\prime}$ & & 0.20 & \\
\hline Disagreement between both detectors, $\%$ & $\mathrm{~A}_{4}^{\prime}$ & & 0.00 & \\
\hline Pileup uncertainty (from model), $\%$ & $\mathrm{~B}_{4}^{\prime}$ & & 0.20 & \\
\hline Code uncertainty, $\%$ & $\mathrm{~B}_{5}^{\prime}$ & & 0.20 & \\
\hline Rutherford cross-section (screening uncertainty), $\%$ & $\mathrm{~B}_{6}^{\prime}$ & & 0.27 & \\
\hline Si stopping power (uncertainty on the a-Si yield), $\%$ & $\mathrm{~A}_{5}^{\prime}$ & & - & \\
\hline Si stopping power (uncertainty on the implant counts), $\%$ & $\mathrm{~A}_{6}^{\prime}$ & & - & \\
\hline Combined extra systematic uncertainty, $\%$ & $\mathrm{U}_{3}^{\prime}$ & & 0.44 & \\
\hline Total combined standard uncertainty (accuracy), $\%$ & $\mathrm{U}_{4}^{\prime}$ & & 0.52 & \\
\hline
\end{tabular}


Table 3: Experimental conditions used for the Sb-CRM and the As-implant samples analysis.

\begin{tabular}{cccccc}
\hline Sample & Ion beam & $\begin{array}{c}\text { Ion beam } \\
\text { energy } \\
(\mathrm{MeV})\end{array}$ & $\begin{array}{c}\text { Ion beam } \\
\text { intensity } \\
(\mathrm{nA})\end{array}$ & $\begin{array}{c}\text { Total } \\
\text { collected } \\
\text { charge }(\mu \mathrm{C})\end{array}$ & $\begin{array}{c}\text { Date of } \\
\text { measurement }\end{array}$ \\
\hline Sb-implant CRM & ${ }^{1} \mathrm{H}^{+}$ & 3.0 & 2 & 190 & Jan 13 \\
Sb-implant CRM & ${ }^{4} \mathrm{He}^{+}$ & 1.5 & 15 & 300 & Jun 13 \\
Sb-implant CRM & ${ }^{7} \mathrm{Li}^{2+}$ & 4.0 & 2 & 10 & Oct 12 \\
As-implant & ${ }^{4} \mathrm{He}^{+}$ & 1.5 & 25 & 850 & Feb 11 \\
As-implant & ${ }^{4} \mathrm{He}^{+}$ & 1.5 & 7 & 10 & Jun 12 \\
As-implant & ${ }^{4} \mathrm{He}^{+}$ & 1.5 & 5 & 30 & Jul 12 \\
As-implant & ${ }^{4} \mathrm{He}^{+}$ & 1.5 & 14 & 29 & Jun 13 \\
As-implant & ${ }^{4} \mathrm{He}^{+}$ & 1.5 & 13 & 27 & Jun 13 \\
As-implant & ${ }^{4} \mathrm{He}^{+}$ & 1.5 & 13 & 45 & Jul 13 \\
As-implant & ${ }^{4} \mathrm{He}^{+}$ & 1.5 & 12 & 40 & Jul 13 \\
As-implant & ${ }^{7} \mathrm{Li}^{2+}$ & 4.0 & 2 & 23 & Oct 12 \\
As-implant & ${ }^{12} \mathrm{C}^{4+}$ & 9.6 & 5 & 25 & Mar 12 \\
As-implant & ${ }^{12} \mathrm{C}^{4+}$ & 9.6 & 52 & \\
\hline
\end{tabular}


Table 4: Summary of the results obtained with various ion beams for Sb-CRM and As-implants.

\begin{tabular}{|c|c|c|c|c|c|}
\hline Ion beam & Target & $\begin{array}{c}\text { Date of } \\
\text { measurement }\end{array}$ & $\begin{array}{l}\text { Measured } \\
\text { fluence } \\
\left(10^{15} \mathrm{~cm}^{-2}\right)\end{array}$ & $\begin{array}{c}\text { Total combined } \\
\text { uncertainty } \\
U_{4}^{\prime} \text { or } U_{4} \\
(\%)\end{array}$ & $\begin{array}{l}\text { Stopping power } \\
\text { factor } \\
\text { uncertainty } \\
(\%)\end{array}$ \\
\hline $\begin{array}{c}3.0 \mathrm{MeV} \\
{ }^{1} \mathrm{H}^{+}\end{array}$ & Sb-CRM & Jan 13 & 41.0 & 1.2 & - \\
\hline $\begin{array}{l}\text { 1.5 MeV } \\
{ }^{4} \mathrm{He}^{+}\end{array}$ & Sb-CRM & Jun 13 & 48.0 & 0.56 & 0.8 \\
\hline $\begin{array}{l}\text { 4.0 MeV } \\
{ }^{7} \mathrm{Li}^{2+}\end{array}$ & Sb-CRM & Oct 12 & 48.4 & 0.82 & 1.0 \\
\hline $\begin{array}{l}\text { 1.5 MeV } \\
{ }^{4} \mathrm{He}^{+}\end{array}$ & As-implant & Feb 11 & 4.632 & 1.09 & 0.8 \\
\hline $\begin{array}{l}\text { 1.5 MeV } \\
{ }^{4} \mathrm{He}^{+}\end{array}$ & As-implant & Jun 12 & 4.699 & 1.55 & 0.8 \\
\hline $\begin{array}{c}1.5 \mathrm{MeV} \\
{ }^{4} \mathrm{He}^{+}\end{array}$ & As-implant & Jul 12 & 4.673 & 1.17 & 0.8 \\
\hline $\begin{array}{l}\text { 1.5 MeV } \\
{ }^{4} \mathrm{He}^{+}\end{array}$ & As-implant & Jun 13 & 4.680 & 1.39 & 0.8 \\
\hline $\begin{array}{c}1.5 \mathrm{MeV} \\
{ }^{4} \mathrm{He}^{+}\end{array}$ & As-implant & Jun 13 & 4.574 & 1.40 & 0.8 \\
\hline $\begin{array}{c}1.5 \mathrm{MeV} \\
{ }^{4} \mathrm{He}^{+}\end{array}$ & As-implant & Jul 13 & 4.648 & 1.08 & 0.8 \\
\hline $\begin{array}{l}\text { 1.5 MeV } \\
{ }^{4} \mathrm{He}^{+}\end{array}$ & As-implant & Jul 13 & 4.635 & 1.10 & 0.8 \\
\hline $\begin{array}{l}\text { 4.0 MeV } \\
{ }^{7} \mathrm{Li}^{2+}\end{array}$ & As-implant & Oct 12 & 4.595 & 1.92 & 1.0 \\
\hline $\begin{array}{l}9.6 \mathrm{MeV} \\
{ }^{12} \mathrm{C}^{4+}\end{array}$ & As-implant & Mar 12 & 4.626 & 3.22 & 3.0 \\
\hline $\begin{array}{l}9.6 \mathrm{MeV} \\
{ }^{12} \mathrm{C}^{4+}\end{array}$ & As-implant & Mar 12 & 4.721 & 3.23 & 3.0 \\
\hline
\end{tabular}

The uncertainty on the stopping power factor used is traceable to the CRM for all cases except for the C-beam analysis, for which it is traceable to Helmut Paul's compilation ${ }^{49}$. The weighted average for the measurements of the As implant fluence with a He beam is $(4.647 \pm 0.043) \times 10^{15} \mathrm{As} / \mathrm{cm}^{2}$. 


\section{Figure captions}

Figure 1: RBS energy spectrum for Det B and from (nominally) $5 \times 10^{15} \mathrm{~cm}^{-2}$ As implant at $80 \mathrm{keV}$ into silicon, together with a (nominally) $3 \times 10^{15} \mathrm{~cm}^{-2} \mathrm{Ar}$ implant at $150 \mathrm{keV}$. The surface signal positions for $\mathrm{Si}, \mathrm{Ar}$ and As are shown together with the area $A_{A s}$ of the arsenic signal (see Eq.1) and the surface yield $Y_{0, S i}$ of the $\mathrm{Si}$ substrate signal (see Eq.3). The beam is $1.5 \mathrm{MeV} \mathrm{He}^{+}$and the channel widths are about $3 \mathrm{keV} / \mathrm{channel}$. The $\mathrm{Si}$ signal is high in the surface (amorphised) region and low in the deeper (crystalline) region in which the beam is channelling. The inset shows the As depth profiles derived from the spectra recorded by Det A (red) and Det B (blue). The apparent yield mismatch for channel numbers $>300$ is misleading: the number of counts in this region is the same for data and fit because there are many channels with no counts.

Figure 2: As-implant fluences measured by a $1.5 \mathrm{MeV}{ }^{4} \mathrm{He}^{+}$beam over a two year period. The error bars are given as the "reduced uncertainty" $\left(U_{5}, \mathrm{k}=1\right.$; see Table 1$)$. The weighted mean of the measurements is calculated using $W_{i}=1 / U_{5}^{i}$.

Figure 3: As-impant fluences measured by a $4.0 \mathrm{MeV}^{7} \mathrm{Li}^{2+}$ or $9.6 \mathrm{MeV}{ }^{12} \mathrm{C}^{4+}$ beams. The weighted mean of $1.5 \mathrm{MeV}^{4} \mathrm{He}^{+}$measurements is also shown for comparison. The error bars are given as the total combined standard uncertainty $\left(U_{4} ; \mathrm{k}=1\right)$ and are dominated by counting statistics for the lithium beam and by the stopping power uncertainty (from the databases in the literature) for the carbon beams. 


\section{Figures}

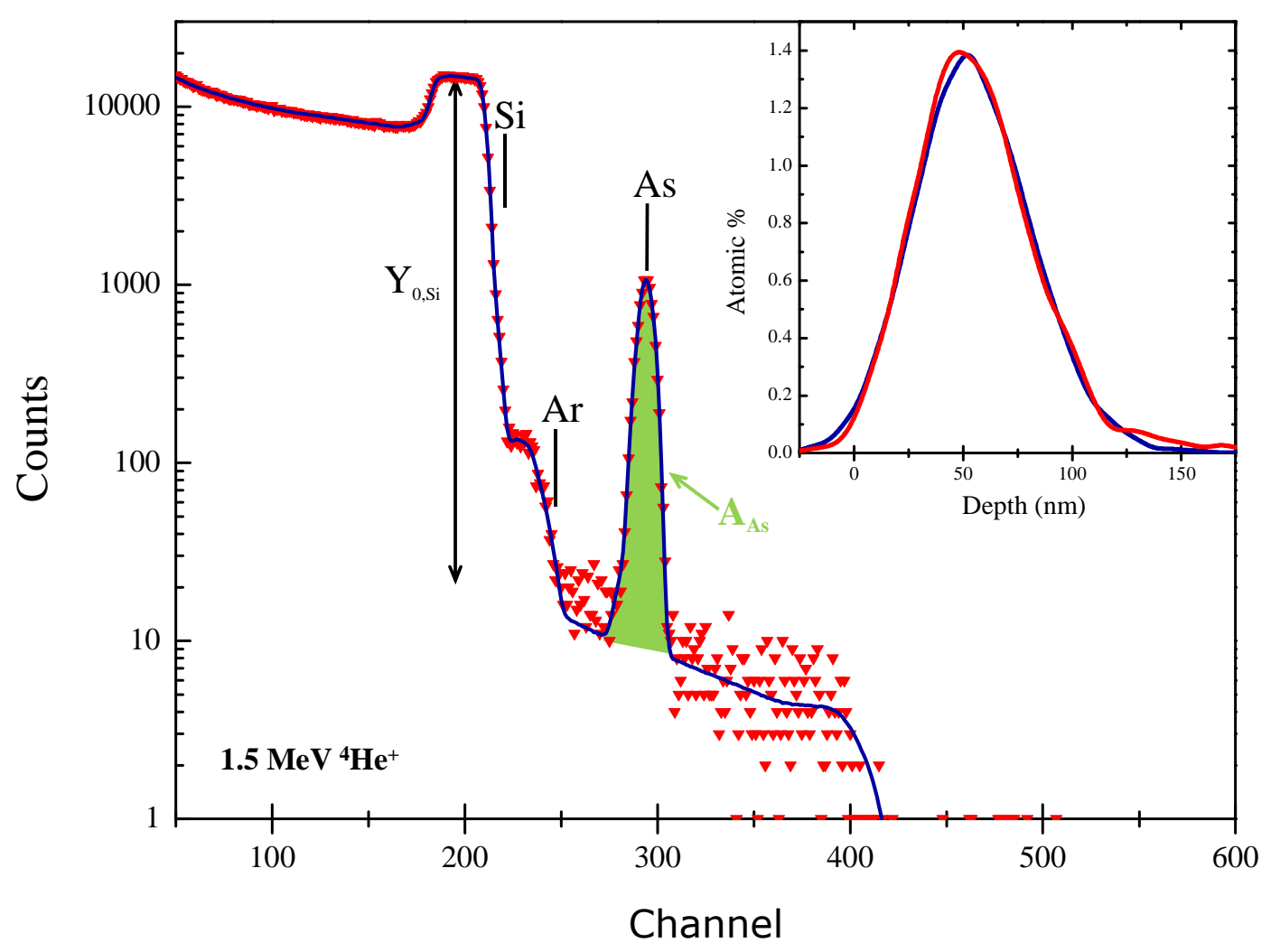

Figure 1

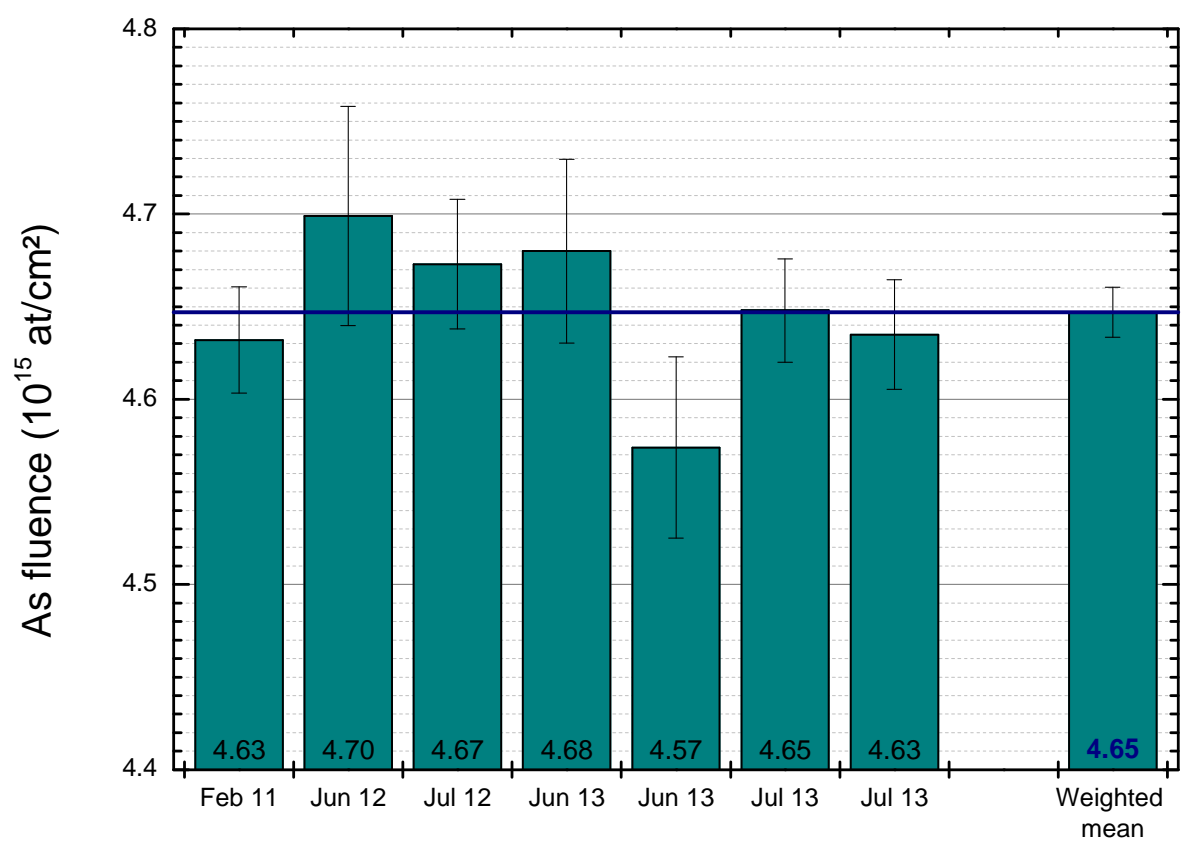

Figure 2 


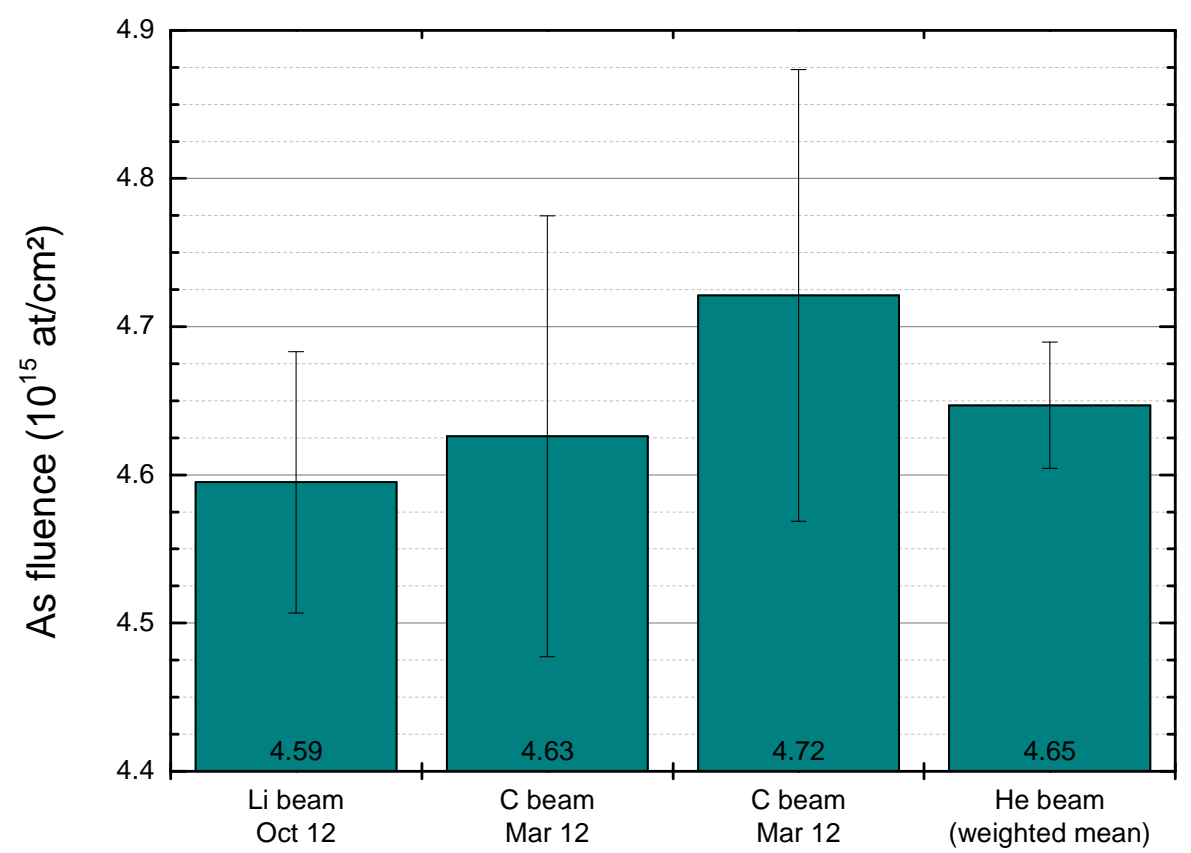

Figure 3 